\title{
El análisis coste-efectividad: por qué y cómo
}

\author{
The cost-effectiveness analysis: Why and how
}

Para que el acceso a los servicios sanitarios dependa de la necesidad clínica y sanitaria, la práctica totalidad de los países desarrollados del mundo optan por su financiación pública. Lo que parece manifestar unos valores de equidad tiene también una justificación en términos de eficiencia, dado que, si se quiere obtener la máxima cantidad y calidad de vida con los recursos disponibles, hay que dejar las extravagancias de lado para centrarse en lo clínicamente efectivo. Siempre cabrá satisfacer caprichos según la capacidad y voluntad de pagar individualmente por los mismos.

Por otra parte, cuando la forma de abordar tanto la innovación como el envejecimiento continuará modulando el ritmo de crecimiento del porcentaje de la riqueza producida por un país que se dedica a servicios sanitarios, ya no basta el criterio de efectividad. Hay que incorporar la consideración de los costes sociales para optar por los servicios menos costosos entre los efectivos. $Y$ no es una cuestión de ahorrar dinero, sino de evitar muertes y sufrimientos innecesarios. Por ejemplo, si en España el lapatinib + capecitabina, en segunda línea para el cáncer de mama, supone $732.000 €$ por año, del orden de 1,5 a 2 millones de euros por año de vida ajustado por calidad (AVAC) (1), el problema no es que dos millones sean mucho dinero sino que se están perdiendo unos 150-200 AVACs en el mejor uso alternativo de esos recursos (lo que se llama coste de oportunidad). Como dice Espriu, a veces hay que sacrificar a un hombre por un pueblo, pero nunca un pueblo debe ser sacrificado por un hombre. $Y$ se pierden 150-200 suponiendo simplemente que el mejor uso alternativo es aproximadamente el coste promedio que supone obtener un AVAC en España (2) o en el Reino Unido (3). También en clínica, hace mucho que Cochrane escribió que no se trata de manejar cada caso individual hasta el límite de lo imposible, pues, como sentenció Donabedian, la eficiencia es la marca del virtuosismo en Medicina.

En España, y en todo el Mezzogiorno europeo, la situación refuerza el clamor por la sensatez. Los datos del Ministerio de Sanidad señalan que entre 2009 y 2013 el gasto sanitario público tuvo una caída del $13 \% \ldots$, a lo que debe añadirse que toca devolver lo que debemos (incluyendo lo despilfarrado en aeropuertos sin aviones, AVEs sin pasajeros o inflando el terciarismo tanto sanitario como universitario). Más aún, la actualización del Programa de Estabilidad 2015-18 que el Reino de España ha comprometido con la Unión Europea implica reducir el gasto sanitario público del 6\% en 2013 al 5,3\% del PIB en 2018. Es cierto que puede esperarse un crecimiento del denominador PIB, incluso una renegociación, pero, en estos momentos, el único componente del gasto sanitario que tiene asegurado un crecimiento similar al del PIB son los productos farmacéuticos innovadores, definidos como los que no sean genéricos, según el pacto firmado entre el Gobierno de España y Farmaindustria en noviembre del 2015. 
No existe ninguna contradicción entre consolidar una conquista de la humanidad como el Estado del Bienestar (EB) y disponer de una economía dinámica y puntera. Suecia, Noruega, Finlandia, Dinamarca e Islandia hace décadas que lo vienen demostrando. Nuestros sistemas del bienestar también deberían huir de las rémoras que los plagan (no tanto subsidiar el no trabajar, como fomentar las políticas activas de empleo y ocupación, impuestos más progresivos...) e imitar a los nórdicos (4). Así, por ejemplo, a la hora de escoger entre el 'para todos' y el 'todo', la preferencia europea por la universalidad exige una adecuada definición de la cartera de servicios que se financia públicamente. De hecho, una cartera que responda tanto a criterios científicos de coste-efectividad como a preferencias sociales constituye el auténtico factor de sostenibilidad de la componente sanitaria del EB. Para no dualizar la sociedad entre aquellos que podrían pagar por cualquier innovación, con independencia de su utilidad, y la gran mayoría que quedaría excluida, hace falta que la introducción, mantenimiento y retirada de tecnología responda a criterios de coste-efectividad.

El análisis coste-efectividad (ACE), primero, proporciona las bases para unas políticas de precios que estimulen la innovación socialmente interesante y permitan una ciudadanía satisfecha con sus servicios sanitarios financiados públicamente, que respalda con su voto. $Y$, segundo, garantiza la sostenibilidad financiera de la componente sanitaria del EB por la vía de su 'deseabilidad'.

La respuesta hasta ahora ha sido del todo insuficiente. Pese a que el ACE está formalmente introducido en España desde la Ley del Medicamento de 1990, en la práctica no se ha incorporado todavía. Así, en el dictamen -no vinculante- de la Comisión Nacional de los Mercados y la Competencia (CNMC) de noviembre 2015 (5), se efectúa un demoledor informe sobre el Proyecto de Real Decreto por el que se regula la financiación y fijación de precios de medicamentos y productos sanitarios y su inclusión en la prestación farmacéutica del Sistema Nacional de Salud. En corto: parecemos renunciar al factor de sostenibilidad de la componente sanitaria del EB. En largo: excesiva discrecionalidad de la Administración, mantenimiento del déficit de transparencia existente y no desarrollo del principio de coste-efectividad, garantía (junto con la consideración del impacto presupuestario) de que el coste de la prestación (farmacéutica en este caso) se vea compensando por los beneficios que reporta a la salud de los ciudadanos, tal como viene sucediendo en los países europeos de referencia. Debe recordarse que los informes de los organismos reguladores como la CNMC permiten orientar una regulación por parte del Estado que evite los defectos del capitalismo -monopolio, amiguismo-y potencie sus virtudes: competencia e innovación.

\section{Entrando en materia}

¿Qué es el ACE? La evaluación de las tecnologías médicas consiste en el análisis de sus costes y consecuencias. La forma como se operacionalizan y miden las consecuencias conforma tres tipos de evaluación. Su medición en términos monetarios (por ej., coste de una enfermedad evitada con la vacunación) define el análisis de coste-beneficio (indica si vale más o no de lo cuesta en dichos términos). Cuando se expresan en unidades de efectividad (aumento de la supervivencia), nos encontramos con un ACE. Si la medida de efectividad se ajusta (este año de vida adicional se corrige) conforme a una escala de calidad de vida (de 0 a 1 , siendo $0=$ muerte y 1 = perfecta salud; es distinto vivir un año adicional muy discapacitado que pudiendo realizar actividades de la vida diaria en condiciones aceptables), se convierte en un análisis de costeutilidad (ACU) (6). Se ha convertido en (mala) costumbre llamar también a este último de coste-efectividad. 


\section{Por qué el ACE}

Según varios análisis recientes publicados, del total de nuevos medicamentos autorizados desde mediados de los ochenta (incluidos los de síntesis química, los biológicos y los biosimilares), es baja la fracción de aquellos con alto valor terapéutico añadido, es decir, con eficacia y seguridad relativas (incrementales, respecto a los disponibles) marcadas (7).

Las agencias reguladoras de la mayoría de países imponen tres requisitos (barreras) para autorizar un medicamento: eficacia, seguridad y calidad. Pero en algunos no todos los autorizados se financian. Los presupuestos son limitados, no se puede financiar todo y cualquier decisión de financiación tiene su coste de oportunidad. Hay que maximizar la salud que obtiene la sociedad con los recursos disponibles, y para ello algunos países usan como regla para decidir si financian o no una nueva tecnología la razón de coste-efectividad (la cuarta barrera): con salvedades, entran en cobertura pública los medicamentos cuya razón de coste-efectividad es igual o menor de cierta cifra (el umbral de coste-efectividad) y se excluyen aquellos con razón de coste-efectividad mayor. Tal como se estima su valor, este umbral expresa la disposición a pagar de una determinada sociedad, cuánto quiere o puede pagar por un AVAC ganado (en el Reino Unido se establece entre 20.000-30.000 libras, unos 30.00040.000 euros por AVAC ganado) (8). (Nótese que, a diferencia del ACE -la efectividad de diferentes tecnologías se expresa de forma distinta-, el ACU, al tener como medida de consecuencias un único denominador común, los AVAC, permite comparar tecnologías diferentes.)

Para afrontar dichas restricciones y la discrepancia en aumento entre el precio (elevadísimo en algunos casos) y el valor terapéutico incremental (bajo) de nuevos medicamentos, en los últimos años los financiadores de un número creciente de países están adoptando paulatinamente más medidas: priorizar la cobertura de aquellas tecnología autorizadas con mayor valor terapéutico añadido y fijar sus precios conforme a dicho valor (value-based pricing) (8).

Esto constituye, en primer lugar, una señal para la industria a canalizar su capacidad innovadora hacia tecnologías con mayor valor añadido y obliga al "buen" regulador a "redirigir" sus incentivos a la industria hacia esta innovación incremental. En segundo lugar, como el regulador autoriza o no y el financiador paga o no lo que se autoriza, se engruesa la nómina de países que armonizan las discrepancias entre los criterios de autorización del primero (la EMA, la FDA, los de Canadá, Japón, Singapur, Australia o Nueva Zelanda, entre otros) y los de fijación de precios (cuánto se paga) y cobertura (para quién) del segundo (9).

Más aún, en algunos países desde no hace mucho reguladores y financiadores añaden a los fabricantes como requisito adicional a la cuarta barrera el análisis de su impacto presupuestario: la estimación del impacto en el gasto sanitario público que cabe esperar de la adopción y difusión de una nueva tecnología en un sistema de salud determinado, atendiendo a sus restricciones presupuestarias y al conjunto de prestaciones incluidas en su cartera de servicios durante un período acotado. Hay un buen ejemplo en España (10).

En suma, la regulación vigente está cambiando y cambiará más. Desde la perspectiva de la sociedad (la de todos nosotros), si queremos maximizar nuestra salud con los recursos que tenemos, hemos de ser eficientes, para lo cual la incorporación y retirada de tecnologías en la cartera de servicios debe atender a su valor incremental y su precio, reflejar la magnitud de dicho incremento y nuestra disposición a pagar (preferencia). 


\section{Cómo se hace}

EI AVAC es una medida de salud que países avanzados utilizan en la toma de decisiones de financiación. La regla para decidir es financiar públicamente las tecnologías médicas que producen la mayor ganancia en salud (más AVACs) con un coste dado.

Estas decisiones las basan fundamentalmente en la siguiente información. Primero, la razón de coste-efectividad incremental: la razón que resulta de dividir la diferencia de costes entre el nuevo medicamento y su alternativa disponible por la diferencia entre sus AVACs (coste por AVAC ganado). Sin embargo, a semejanza de cualquier otra estimación (media, porcentaje), siempre hay incertidumbre asociada con las estimaciones de costes y efectividades o utilidades. Como la razón de coste-efectividad incremental es una razón, es complejo calcular sus intervalos de confianza para expresar dicha incertidumbre. En su lugar, se utilizan métodos estadísticos que producen diferentes valores posibles (estimados) de la razón de costeefectividad incremental. Segundo, la llamada curva de aceptabilidad. Con los valores de la razón de coste-efectividad incremental (del umbral) así estimados, esta curva indica gráficamente cuál es la probabilidad $(20,40,70,90 \% . .$.$) de que una nueva tecnología sea$ coste-efectiva (menor o igual al umbral fijado ) comparada con su alternativa disponible para cada valor del umbral $(20.000,30.000,40.000$ por AVAC ganado) (11) (figura 1 ).

No obstante, considerada la labilidad de las preferencias sociales por el impacto de las emociones y su incongruencia temporal, junto con la relativa ignorancia sobre cómo se generan tales preferencias y cómo se alteran en función del marco en que se formula el problema y los importantes límites de nuestra racionalidad, conviene no fiarlo todo a la técnica y fortalecer los procedimientos que permiten un establecimiento de prioridades más democrático. La correcta medición de los valores y las preferencias sociales puede también expresarse mediante la participación responsable de los ciudadanos implicados, que somos todos cuando se trata de formular prioridades sobre la asignación de recursos financiados públicamente (no sólo el segmento beneficiario) (12).

Desde hace poco tiempo, algunas sociedades científicas han empezado a incorporar en sus recomendaciones para evaluar nuevos medicamentos, además del análisis de la relación beneficio-riesgo clínicos, el análisis de coste-efectividad, como el American College of Cardiology y la European Society of Medical Oncology. La Sociedad Española de Cardiología no es ajena a esta tendencia (sí pionera en España), como muestran los objetivos de InnovaSEC (13).

La información que aportan la razón de coste-efectividad incremental y el impacto presupuestario se complementa. Como el número de pacientes que van a beneficiarse de la nueva tecnología importa, si son pocos, el análisis de impacto presupuestario de una nueva tecnología cuya razón de coste-efectividad incremental sea muy alta (alto coste/baja efectividad como lapatinib) será bajo. Si la razón de coste-efectividad incremental es baja y han de tratarse muchos, su impacto presupuestario será alto. No debe olvidarse que, tras introducirla, al aumentar los pacientes tratados, también lo hace la información que se va acopiando sobre su efectividad y seguridad, y con el tiempo y la aparición de nuevas alternativas terapéuticas y los efectos de sustitución, los precios cambian. Por ello, las razón de coste-efectividad incremental tienen que interpretarse desde una perspectiva dinámica, del mismo modo que cuando las comparamos entre países (los costes varían entre ellos).

\section{El ACE y los biológicos en cardiología: ¿previsión de una nueva fuerte marejada?}

En los últimos dos años hemos asistido a escala internacional a los numerosos problemas y controversias desatadas en torno a los nuevos medicamentos contra la hepatitis C: alta 
eficacia estimada, de momento, a corto-medio plazo, pero elevados costes, muchos pacientes por tratar, marcadas diferencias en los precios entre países, muy alto impacto presupuestario, y variabilidad pronunciada en la forma como los reguladores y financiadores de los países han decidido qué pacientes quedan cubiertos y la forma de financiar los tratamientos. De esta variabilidad resultan ineficiencias, más en unos países que en otros. El ACE es una - no la única - herramienta que ayuda sobremanera a mejorar la información disponible para tomar estas difíciles decisiones y, por consiguiente, para reducir el menoscabo de la eficiencia social que produce decidir con información insuficiente o deficiente.

Es deseable que no ocurra lo mismo con la posible fuerte marejada que puedan desencadenar los inhibidores de la PCSK9 (notable descenso de la LDL en sus indicaciones, altos costes e indicaciones concretas que pueden incumplirse): los ya autorizados alirocumab y evolocumab, el bococizumab, que se encuentra en fase III, y otra nueva molécula de síntesis química inhibidora también de la PCSK9 y una vacuna contra esta misma enzima, que se espera entren en ensayos clínicos este año (14). Aproximándonos a lo que hacen otros países avanzados en materia de regulación y financiación, y aplicando los principios y herramientas aquí descritos, podríamos reducir el alto riesgo de incurrir en ineficiencias que a la postre todos pagamos.

\section{Algunas conclusiones}

Como se ha afirmado sabiamente, verdadera innovación, accesibilidad y sostenibilidad [añadimos solvencia] de los sistemas sanitarios son engranajes del mismo mecanismo (15) . Aquellos con amplia cobertura pública deben consolidar y mantener a largo plazo una buena regulación $(I+D$, autorización, fijación de precio, cobertura, vigilancia e información postcomercialización, des y reinversión). En España tenemos capacidad técnica para hacerlo, podríamos crear la agencia, independiente y vinculante, para tomar las decisiones adecuadas (como intenta el NICE), pero no aplicamos todas las reglas de juego conocidas (valor terapéutico añadido, razón de coste-efectividad incremental, impacto presupuestario, reinversión), aún no rendimos cuentas claras, no somos transparentes en lo que hacemos y dejamos de hacer, ni hemos conseguido que en estas decisiones participen todos los afectados por ellas.

La regulación está sometida a profundo escrutinio, se han hecho numerosos cambios regulatorios pre y postcomercialización y hay nuevas propuestas en marcha en algunos países. Hay que convertir una regulación binaria (se autoriza/no se autoriza, se financia/no se financia) en un continuo de fases de obtención de información sobre eficacia, efectividad y seguridad (antes y después de autorizarlas condicionalmente), adaptar oportunamente los estándares de evidencia exigibles a los imperativos de la innovación y, en función de las evidencias generadas progresivamente, favorecer el acceso más temprano a las que muestren eficacia y seguridad, modificar las indicaciones y los criterios de cobertura pública y su pronta retirada del mercado cuando proceda, y adaptar precios en virtud de su valor conocido en cada etapa (9).

El diagnóstico realizado en España sobre nuestros problemas regulatorios y sus consecuencias en la salud pública es más que aceptable. Se han propuesto suficientes medidas estructurales de mejora, que requieren recursos de distinta índole, así como la colaboración de todos los agentes implicados y el apoyo político firme y permanente.

La evaluación tiene mucho de bien público: los resultados que produce podemos utilizarlos todos y su uso por unos no impide que los usen otros. España podría incluso permitirse una moratoria de no incorporación de nueva tecnología durante un período para parecerse a países de mayor riqueza pero, sobre todo, con un EB más consolidado. 


\section{Referencias}

1. Oyaguez I, Frías C, Seguí MA, Gómez-Barrera M, Casado MA, Queralt Gorgas M. Eficiencia de tratamientos oncológicos para tumores sólidos en España. Farm Hosp. 2013;37:240-59.

2. Puig-Junoy J, Merino-Castelló A. Productividad marginal del gasto e innovaciones sanitarias. Resultados empíricos y lecciones para España. En: Puig-Junoy J, LópezCasasnovas, Ortún V (editores). ¿Más recursos para la salud? Barcelona: Masson; 2004:133-54.

3. Claxton K, Martin S, Soares M, Rice N, Spackman E, Hinde S, et al. Methods for the estimation of the National Institute for Health and Care Excellence cost-effectiveness threshold. Health Technol Assess Health Care. 2015;19(14). doi: 10.3310/hta19140.

4. López-Casasnovas G. El bienestar desigual. Barcelona: Península; 2015.

5. Comisión Nacional de los Mercados y la Competencia. IPN/CNMC/023/15 Proyecto de Real Decreto por el que se regula la financiación y fijación de precios de medicamentos y productos sanitarios y su inclusión en la prestación farmacéutica del Sistema Nacional de Salud. (Disponible en www.cnmc.es)

6. Drummond MF, Sculpher MJ, Torrance GW, O'Brien BJ, Stoddart GL. Methods for the economic evaluation of health care programmes. 3rd, ed. New York: Oxford University Press; 2005.

7. Campillo-Artero C. Nuevos medicamentos. ¿Cuánto se innova? Ges Clin Sanit. 2015;17:7-9.

8. Claxton K, Sculpher M, Carroll S. Value-based pricing for pharmaceuticals: Its role, specification and prospects in a newly devolved NHS. York: Centre for Health Economics, University of York; 2011. (CHE Research paper 60.)

9. Eichler HG, Oye K, Baird LG, et al. Adaptive licensing: taking the next step in the evolution of drug approval. Clin Pharmacol Ther. 2012;91:426-37.

10. Puig-Junoy, J, Oliva J, Trapero M, Abellán JM, Brosa M. Guía y recomendaciones para la realización y presentación de evaluaciones económicas y análisis de impacto presupuestario de medicamentos en el ámbito del Catsalut. Barazón de costeefectividadlona: Catsalut; $2014 . \quad$ Disponible en: http://catsalut.gencat.cat/web/.content/minisite/catsalut/proveidors_professionals/medi caments_farmacia/farmaeconomica/caeip/documents/gaeip_publica_castellano_octubre2 014_catsalut.pdf

11. Oliva J, Campillo-Artero C, Hidalgo A, Sanz A. Evaluación económica en oncología. Madrid: Entheos; 2012.

12. González López-Valcárcel B, Ortún V. Pals don't evaluate pals...or do they? Rev Esp Salud Pública. 2015;89:119-23.

13. Sampietro-Colom L, Brugada-Terradellas J, González-Juanatey JR. Introdución de innovaciones en el área de la patología cardiaca en España: InnovaSEC. Rev Esp Cardiol. 2015;68:834-7.

14. Mullard A. PCSK9 inhibitors are go. Nat Rev Drug Discover. 2015;14:593.

15. Peiró S. La incorporación de nuevos medicamentos al SNS: Innovación, accesibilidad y sostenibilidad. Ges Clin Sanit. 2015;17:5-6. 
Figura 1. Curva de aceptabilidad.

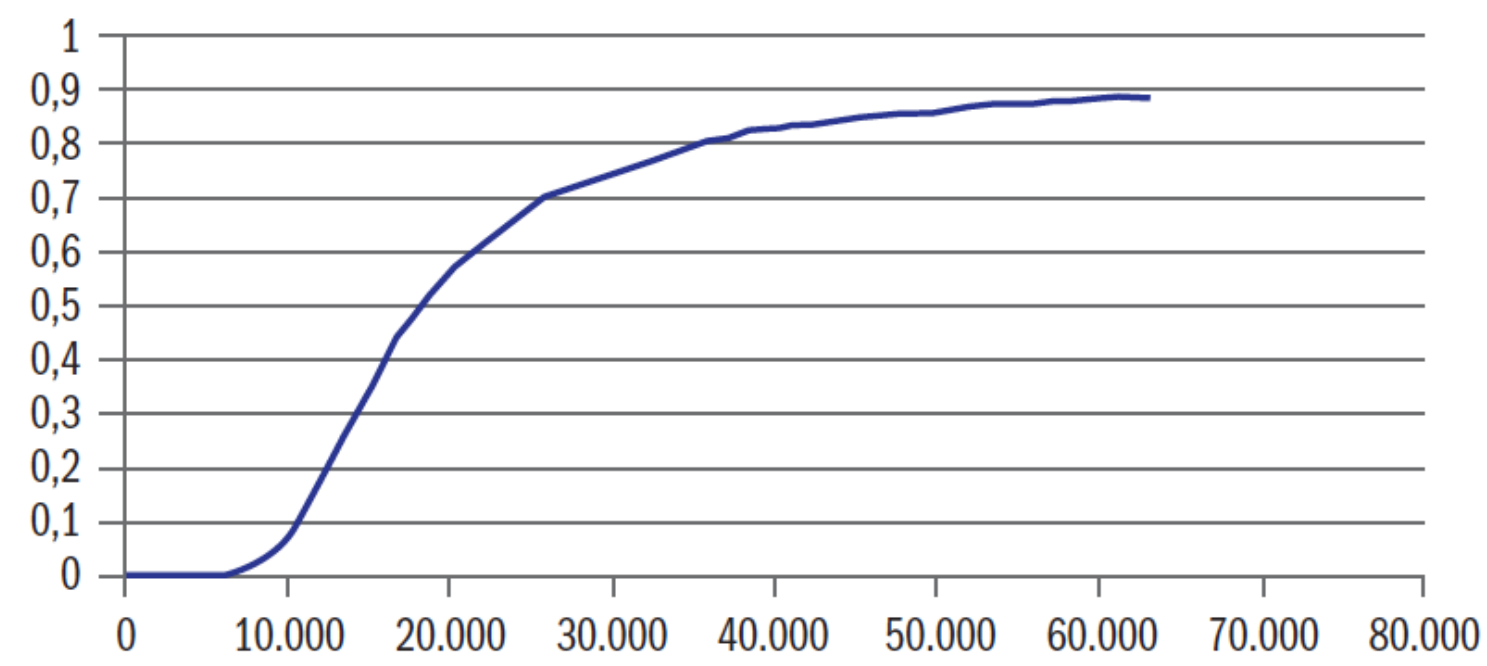

Leyenda eje de la y: Probabilidad de que la tecnología sea coste-efectiva comparada con la alternativa

Leyenda eje de la x: Razón (umbral) de coste-efectividad incremental (por año de vida ajustado por calidad ganado) en euros 


\section{REVISTA ESPAÑOLA DE CARDIOLOG A}

\section{FORMULARIO UNIFORME DE DECLARACIÓN DE POSIBLES CONFLICTOS DE INTERESES}

\section{Sección 1. Identificación}

Nombre: Carlos

Apellidos: Campillo-Artero

Fecha: 7 de enero de 2016

¿Es Ud. el autor de correspondencia? $\square$ Sí $\square$ No

Nombre del autor de correspondencia:Vicente Ortún Rubio

Título del manuscrito:El análisis coste-efectividad: por qué y cómo

Número de referencia del manuscrito (si lo conoce):

\section{Sección 2. Información sobre la financiación del trabajo considerado para publicación}

Usted o su institución ¿han recibido en algún momento pagos por o ayudas para cualquier aspecto del trabajo presentado? (la ayuda incluye, pero no se limita a, becas, diseño del estudio, comité de control de datos, preparación del manuscrito, análisis estadístico, etc.)

Cumplimente cada fila marcando "No" o incorporando la información necesaria.

\begin{tabular}{|l|l|l|l|l|l|}
\hline Tipo & No & $\begin{array}{l}\text { Pago a } \\
\text { Ud. }\end{array}$ & $\begin{array}{l}\text { Pago a su } \\
\text { institución* }\end{array}$ & $\begin{array}{l}\text { Nombre de } \\
\text { la entidad }\end{array}$ & Comentarios $^{\star *}$ \\
\hline Becas & $x$ & & & & \\
\hline Honorarios por consultoría & $x$ & & & & \\
\hline $\begin{array}{l}\text { Remuneración por desplazamiento a } \\
\text { reuniones relacionadas con el presente } \\
\text { trabajo o de otro tipo }\end{array}$ & $x$ & & & & \\
\hline $\begin{array}{l}\text { Remuneración por participación en } \\
\text { revisiones; ej., comités de control de } \\
\text { datos, análisis estadístico, comités de } \\
\text { objetivos y similares }\end{array}$ & $x$ & & & & \\
\hline $\begin{array}{l}\text { Remuneración por redacción o revisión } \\
\text { del manuscrito }\end{array}$ & $x$ & & & & \\
\hline $\begin{array}{l}\text { Retribución en especie, por ejemplo, } \\
\text { redacción, medicamentos o } \\
\text { equipamiento, apoyo administrativo }\end{array}$ & $x$ & & & & \\
\hline \begin{tabular}{l} 
Otros \\
\hline
\end{tabular} & $x$ & & & & \\
\hline
\end{tabular}

* Pago a su institución recibido por la dedicación/esfuerzo del firmante.

** Utilice esta sección para aportar la información pertinente. 


\section{Sección 3. Información sobre relaciones económicas pertinentes que no sean las relativas al trabajo presentado}

Marque las casillas apropiadas para indicar si tiene alguna relación económica (independientemente de la cantidad) con cualquier entidad que tenga interés relacionado con el trabajo presentado. Utilice la columna de comentarios para añadir cualquier información sobre compensación que, en su opinión, deba conocer un posible lector o editor.

Informe sobre cualquier relación que existiera durante los 36 meses anteriores a la presentación de su trabajo.

\begin{tabular}{|c|c|c|c|c|c|}
\hline Tipo de relación & No & $\begin{array}{l}\text { Pago a } \\
\text { Ud. }\end{array}$ & $\begin{array}{l}\text { Pago a su } \\
\text { institución* }\end{array}$ & Entidad & Comentarios \\
\hline Miembro de Consejo & $x$ & & & & \\
\hline Consultoría & $x$ & & & & \\
\hline Empleo & $x$ & & & & \\
\hline Testimonio de experto & & $x$ & & $\begin{array}{l}\text { Sociedades } \\
\text { científicas, } \\
\text { OMS }\end{array}$ & \\
\hline Becas/becas pendientes & $\mathrm{x}$ & & & & \\
\hline $\begin{array}{l}\text { Remuneración por } \\
\text { desarrollo de ponencias, } \\
\text { incluido servicio en } \\
\text { gabinetes de ponentes }\end{array}$ & & $x$ & & $\begin{array}{l}\text { Sociedades } \\
\text { científicas, } \\
\text { Universidade } \\
\text { s, Merck, } \\
\text { Pfizer, } \\
\text { Novartis, } \\
\text { Sanidad, } \\
\text { Comunicació } \\
\text { n y Gestión } \\
\text { del } \\
\text { conocimiento }\end{array}$ & \\
\hline $\begin{array}{l}\text { Remuneración por } \\
\text { preparación de } \\
\text { manuscritos }\end{array}$ & & $x$ & & $\begin{array}{l}\text { Fundación } \\
\text { Gaspar Casal }\end{array}$ & \\
\hline $\begin{array}{l}\text { Patentes (en proyecto, } \\
\text { pendientes o emitidas) }\end{array}$ & $x$ & & & & \\
\hline Regalías (royalties) & $x$ & & & & \\
\hline $\begin{array}{l}\text { Remuneración por } \\
\text { desarrollo de } \\
\text { presentaciones } \\
\text { educativas }\end{array}$ & & $x$ & & $\begin{array}{l}\text { Sociedades } \\
\text { científicas, } \\
\text { OMS, } \\
\text { Unversidades }\end{array}$ & \\
\hline $\begin{array}{l}\text { Acciones/opción a } \\
\text { acciones }\end{array}$ & $x$ & & & & \\
\hline $\begin{array}{l}\text { Gastos de viaje, } \\
\text { alojamiento y/o } \\
\text { asistencia a reuniones } \\
\text { no relacionados con las } \\
\text { actividades mencionadas } \\
\text { anteriormente }{ }^{\star *}\end{array}$ & $x$ & & & & \\
\hline $\begin{array}{l}\text { Otros (en caso de duda, } \\
\text { declárelo) }\end{array}$ & $x$ & & & & \\
\hline
\end{tabular}

* Pago a su institución recibido por la dedicación/esfuerzo del firmante.

** Por ejemplo, si informa de actividad de consultoría en la fila "Consultoría", no es necesario que informe de gastos de viaje/alojamiento relacionados con la misma en esta fila. 
¿Hay otras relaciones o actividades susceptibles de ser consideradas como influencia o posible influencia en el contenido de su manuscrito?

\No, no tengo relación, interés, condición o circunstancia que constituya un conflicto de interés potencial $\checkmark$ Sí, existen las siguientes relaciones, condiciones o circunstancias: 


\section{REVISTA ESPAÑOLA DE CARDIOLOG A}

\section{FORMULARIO UNIFORME DE DECLARACIÓN DE POSIBLES CONFLICTOS DE INTERESES}

\section{Sección 1. Identificación}

Nombre: Vicente

Apellidos: Ortún Rubio

Fecha: 7enero2016

¿Es Ud. el autor de correspondencia? $\bigotimes$ Sí $\square$ No

Nombre del autor de correspondencia:

Título del manuscrito:El análisis coste-efectividad: por qué y cómo

Número de referencia del manuscrito (si lo conoce):

\section{Sección 2. Información sobre la financiación del trabajo considerado para publicación}

Usted o su institución ¿han recibido en algún momento pagos por o ayudas para cualquier aspecto del trabajo presentado? (la ayuda incluye, pero no se limita a, becas, diseño del estudio, comité de control de datos, preparación del manuscrito, análisis estadístico, etc.)

Cumplimente cada fila marcando "No" o incorporando la información necesaria.

\begin{tabular}{|l|l|l|l|l|l|}
\hline Tipo & No & $\begin{array}{l}\text { Pago a } \\
\text { Ud. }\end{array}$ & $\begin{array}{l}\text { Pago a su } \\
\text { institución* }\end{array}$ & $\begin{array}{l}\text { Nombre de } \\
\text { la entidad }\end{array}$ & Comentarios $^{\star *}$ \\
\hline Becas & $x$ & & & & \\
\hline Honorarios por consultoría & $x$ & & & & \\
\hline $\begin{array}{l}\text { Remuneración por desplazamiento a } \\
\text { reuniones relacionadas con el presente } \\
\text { trabajo o de otro tipo }\end{array}$ & $x$ & & & & \\
\hline $\begin{array}{l}\text { Remuneración por participación en } \\
\text { revisiones; ej., comités de control de } \\
\text { datos, análisis estadístico, comités de } \\
\text { objetivos y similares }\end{array}$ & $x$ & & & & \\
\hline $\begin{array}{l}\text { Remuneración por redacción o revisión } \\
\text { del manuscrito }\end{array}$ & $x$ & & & & \\
\hline $\begin{array}{l}\text { Retribución en especie, por ejemplo, } \\
\text { redacción, medicamentos o } \\
\text { equipamiento, apoyo administrativo }\end{array}$ & $x$ & & & & \\
\hline \begin{tabular}{l} 
Otros \\
\hline
\end{tabular} & $x$ & & & & \\
\hline
\end{tabular}

* Pago a su institución recibido por la dedicación/esfuerzo del firmante.

** Utilice esta sección para aportar la información pertinente. 


\section{Sección 3. Información sobre relaciones económicas pertinentes que no sean las relativas al trabajo presentado}

Marque las casillas apropiadas para indicar si tiene alguna relación económica (independientemente de la cantidad) con cualquier entidad que tenga interés relacionado con el trabajo presentado. Utilice la columna de comentarios para añadir cualquier información sobre compensación que, en su opinión, deba conocer un posible lector o editor.

Informe sobre cualquier relación que existiera durante los 36 meses anteriores a la presentación de su trabajo.

\begin{tabular}{|c|c|c|c|c|c|}
\hline Tipo de relación & No & $\begin{array}{l}\text { Pago a } \\
\text { Ud. }\end{array}$ & $\begin{array}{l}\text { Pago a su } \\
\text { institución* }\end{array}$ & Entidad & Comentarios \\
\hline Miembro de Consejo & $x$ & & & & \\
\hline Consultoría & $x$ & & & & \\
\hline Empleo & $x$ & & & & \\
\hline Testimonio de experto & & $x$ & & OMS & \\
\hline Becas/becas pendientes & $x$ & & $x$ & $\begin{array}{l}\text { Merck } \\
\text { Foundation, } \\
\text { Whitehouse } \\
\text { Station,New } \\
\text { Jersey.,EEU } \\
\text { U }\end{array}$ & $\begin{array}{l}\text { Program on Pharmaceutical } \\
\text { Policy Issues otorgado en } \\
\text { concurso competitivo a seis } \\
\text { universidades en el mundo, } \\
\text { entre ellas al consorcio } \\
\text { Universidad Carlos III de } \\
\text { Madrid-Universidad Pompeu } \\
\text { Fabra de Barcelona entre los } \\
\text { años } 2000 \text { y 2009. Vicente } \\
\text { Ortún, investigador principal. }\end{array}$ \\
\hline $\begin{array}{l}\text { Remuneración por } \\
\text { desarrollo de ponencias, } \\
\text { incluido servicio en } \\
\text { gabinetes de ponentes }\end{array}$ & & $x$ & $x$ & $\begin{array}{l}\text { Sociedades } \\
\text { científicas, } \\
\text { universidade } \\
\text { s }\end{array}$ & \\
\hline $\begin{array}{l}\text { Remuneración por } \\
\text { preparación de } \\
\text { manuscritos }\end{array}$ & $x$ & & & & \\
\hline $\begin{array}{l}\text { Patentes (en proyecto, } \\
\text { pendientes o emitidas) }\end{array}$ & $x$ & & & & \\
\hline Regalías (royalties) & $x$ & & & & \\
\hline $\begin{array}{l}\text { Remuneración por } \\
\text { desarrollo de } \\
\text { presentaciones } \\
\text { educativas }\end{array}$ & & $x$ & $x$ & $\begin{array}{l}\text { Sociedades } \\
\text { científicas, } \\
\text { universidade } \\
\text { s }\end{array}$ & \\
\hline $\begin{array}{l}\text { Acciones/opción a } \\
\text { acciones }\end{array}$ & $x$ & & & Sanofi & $\begin{array}{l}32.000 € \text { (dentro de una } \\
\text { cartera diversificada) }\end{array}$ \\
\hline $\begin{array}{l}\text { Gastos de viaje, } \\
\text { alojamiento y/o } \\
\text { asistencia a reuniones } \\
\text { no relacionados con las } \\
\text { actividades mencionadas } \\
\text { anteriormente }{ }^{\star *}\end{array}$ & $x$ & & & & \\
\hline $\begin{array}{l}\text { Otros (en caso de duda, } \\
\text { declárelo) }\end{array}$ & $x$ & & & & \\
\hline
\end{tabular}

* Pago a su institución recibido por la dedicación/esfuerzo del firmante.

** Por ejemplo, si informa de actividad de consultoría en la fila "Consultoría", no es necesario que informe de gastos de viaje/alojamiento relacionados con la misma en esta fila. 
¿Hay otras relaciones o actividades susceptibles de ser consideradas como influencia o posible influencia en el contenido de su manuscrito?

\No, no tengo relación, interés, condición o circunstancia que constituya un conflicto de interés potencial $\checkmark$ Sí, existen las siguientes relaciones, condiciones o circunstancias: 
Figura 1. Curva de aceptabilidad.

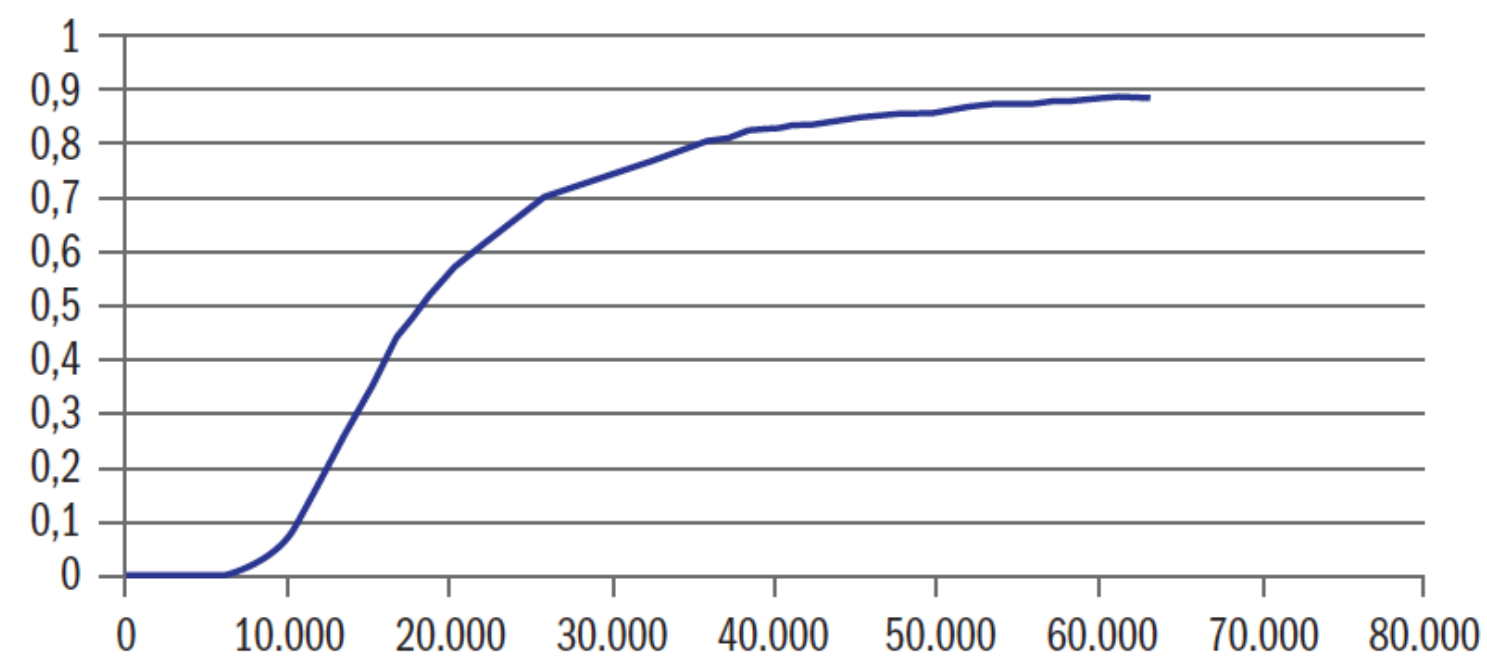

Leyenda eje de la y: Probabilidad de que la tecnología sea coste-efectiva comparada con la alternativa

Leyenda eje de la x: Razón (umbral) de coste-efectividad incremental (por año de vida ajustado por calidad ganado) en euros 